\title{
Dark Matter Search Results from the PandaX-4T Commissioning Run
}

Yue Meng, ${ }^{1,2}$ Zhou Wang, ${ }^{1,2,3}$ Yi Tao, ${ }^{1,2}$ Abdusalam Abdukerim, ${ }^{1}$ Zihao Bo, ${ }^{1}$ Wei Chen, ${ }^{1}$ Xun Chen, ${ }^{1,2}$ Yunhua Chen, ${ }^{4}$ Chen Cheng, ${ }^{5}$ Yunshan Cheng, ${ }^{6,7}$ Xiangyi Cui, ${ }^{3}$ Yingjie Fan, ${ }^{8}$ Deqing Fang, ${ }^{9}$ Changbo Fu, ${ }^{9}$ Mengting Fu, ${ }^{10}$ Lisheng Geng, ${ }_{11,12}$ Karl Giboni, ${ }^{1}$ Linhui Gu, ${ }^{1}$ Xuyuan Guo, ${ }^{4}$ Ke Han, ${ }^{1}$ Changda He, ${ }^{1}$ Jinrong He, ${ }^{4}$ Di Huang, ${ }^{1}$ Yanlin Huang, ${ }^{13}$ Zhou Huang, ${ }^{1}$ Ruquan Hou, ${ }^{2}$ Xiangdong Ji, ${ }^{14}$ Yonglin $\mathrm{Ju},{ }^{15}$ Chenxiang Li, ${ }^{1}$ Mingchuan Li, ${ }^{4}$ Shu Li, ${ }^{15}$ Shuaijie Li, ${ }^{3}$ Qing Lin,${ }^{16,17, *}$ Jianglai Liu, ${ }^{1,3,2,}$ Xiaoying Lu, ${ }^{6,7}$ Lingyin Luo, ${ }^{10}$ Wenbo Ma, ${ }^{1}$ Yugang Ma, ${ }^{9}$

Yajun Mao, ${ }^{10}$ Nasir Shaheed, ${ }^{6,7}$ Xuyang Ning, ${ }^{1}$ Ningchun Qi ${ }^{4}$ Zhicheng Qian, ${ }^{1}$ Xiangxiang Ren,${ }^{6,7}$ Changsong Shang, ${ }^{4}$ Guofang Shen, ${ }^{11}$ Lin Si, ${ }^{1}$ Wenliang Sun, ${ }^{4}$ Andi Tan, ${ }^{14}$ Anqing Wang, ${ }^{6,7}$ Meng Wang, ${ }^{6,7}$ Qiuhong Wang, ${ }^{9}$ Shaobo Wang, ${ }^{1,18}$ Siguang Wang, ${ }^{10}$ Wei Wang, ${ }^{5}$ Xiuli Wang, ${ }^{15}$ Mengmeng Wu, ${ }^{5}$ Weihao Wu, ${ }^{1}$ Jingkai Xia, ${ }^{1}$ Mengjiao Xiao, ${ }^{14}$ Xiang Xiao, ${ }^{5}$ Pengwei Xie, ${ }^{3}$ Binbin Yan, ${ }^{1}$ Xiyu Yan, ${ }^{13}$ Jijun Yang, ${ }_{1}^{1}$ Yong Yang, ${ }^{1}$ Chunxu Yu, ${ }^{8}$ Jumin Yuan, ${ }^{6,7}$ Ying Yuan, ${ }^{1}$ Dan Zhang, ${ }^{14}$ Minzhen Zhang, ${ }^{1}$ Peng Zhang, ${ }^{4}$ Tao Zhang, ${ }^{1}$ Li Zhao, ${ }^{1}$ Qibin Zheng, ${ }^{13}$ Jifang Zhou, ${ }^{4}$ Ning Zhou $\odot,{ }^{1, *}$ Xiaopeng Zhou, ${ }^{11,8}$ and Yong Zhou ${ }^{4}$

(PandaX-4T Collaboration)

${ }^{1}$ School of Physics and Astronomy, Shanghai Jiao Tong University, MOE Key Laboratory for Particle Astrophysics and Cosmology,

Shanghai Key Laboratory for Particle Physics and Cosmology, Shanghai 200240, China

${ }^{2}$ Shanghai Jiao Tong University Sichuan Research Institute, Chengdu 610213, China

${ }^{3}$ Tsung-Dao Lee Institute, Shanghai Jiao Tong University, Shanghai, 200240, China

${ }^{4}$ Yalong River Hydropower Development Company, Limited, 288 Shuanglin Road, Chengdu 610051, China

${ }^{5}$ School of Physics, Sun Yat-Sen University, Guangzhou 510275, China

${ }^{6}$ Research Center for Particle Science and Technology, Institute of Frontier and Interdisciplinary Science, Shandong University, Qingdao 266237, Shandong, China

${ }^{7}$ Key Laboratory of Particle Physics and Particle Irradiation of Ministry of Education, Shandong University, Qingdao 266237, Shandong, China

${ }^{8}$ School of Physics, Nankai University, Tianjin 300071, China

${ }^{9}$ Key Laboratory of Nuclear Physics and Ion-beam Application (MOE), Institute of Modern Physics, Fudan University, Shanghai 200433, China

${ }^{10}$ School of Physics, Peking University, Beijing 100871, China

${ }^{11}$ School of Physics, Beihang University, Beijing 100191, China

${ }^{12}$ International Research Center for Nuclei and Particles in the Cosmos and Beijing Key Laboratory of Advanced Nuclear Materials and Physics, Beihang University, Beijing 100191, China

${ }^{13}$ School of Medical Instrument and Food Engineering, University of Shanghai for Science and Technology, Shanghai 200093, China

${ }^{14}$ Department of Physics, University of Maryland, College Park, Maryland 20742, USA

${ }^{15}$ School of Mechanical Engineering, Shanghai Jiao Tong University, Shanghai 200240, China

${ }^{16}$ State Key Laboratory of Particle Detection and Electronics, University of Science and Technology of China, Hefei 230026, China

${ }^{17}$ Department of Modern Physics, University of Science and Technology of China, Hefei 230026, China

${ }^{18}$ SJTU Paris Elite Institute of Technology, Shanghai Jiao Tong University, Shanghai 200240, China

(Received 3 August 2021; accepted 18 November 2021; published 23 December 2021)

We report the first dark matter search results using the commissioning data from PandaX-4T. Using a time projection chamber with 3.7 tonne of liquid xenon target and an exposure of 0.63 tonne $\cdot$ year, 1058 candidate events are identified within an approximate nuclear recoil energy window between 5 and $100 \mathrm{keV}$. No significant excess over background is observed. Our data set a stringent limit to the dark matter-nucleon spin-independent interactions, with a lowest excluded cross section (90\% C.L.) of $3.8 \times 10^{-47} \mathrm{~cm}^{2}$ at a dark matter mass of $40 \mathrm{GeV} / \mathrm{c}^{2}$.

DOI: 10.1103/PhysRevLett.127.261802

Published by the American Physical Society under the terms of the Creative Commons Attribution 4.0 International license. Further distribution of this work must maintain attribution to the author(s) and the published article's title, journal citation, and DOI. Funded by $\operatorname{SCOAP}$. 
Like ordinary matter, the mysterious dark matter in the Universe may be composed of fundamental particles [1]. The hunt for these particles has been intensively carried out globally using many different particle detectors [2-5]. Dark matter direct detection experiments, typically located deep underground, are particularly sensitive to dark matter within a mass range approximately from $\mathrm{GeV} / c^{2}$ to $100 \mathrm{TeV} / c^{2}$, via the nuclear recoil (NR) of the target nucleus [2]. In recent years, large-scale liquid xenon time projection chambers (TPCs) have spearheaded the detection sensitivity [6-9], and three new experiments with multitonne of targets are ongoing to deepen the search [10-12].

The PandaX experiment, located in the China Jinping Underground Laboratory (CJPL) [13], is dedicated to search for dark matter particles and to study fundamental properties of neutrinos. PandaX-4T [10], with a sensitive target of 3.7 tonne of liquid xenon, is located in the B2 hall of the newly expanded CJPL-II [14]. The detector is placed at the center of an ultrapure water shield in a stainless steel tank with a diameter of $10 \mathrm{~m}$ and a depth of $13 \mathrm{~m}$. The double-vessel cryostat made out of low-background stainless steel [15] contains 5.6 tonne of total liquid xenon, with a 30-1 overflow chamber inside the cryostat for adjusting the liquid level [16]. A cryogenic system containing three independent cold heads is constantly delivering cooling power (580 W at maximum) to liquefy xenon [17]. The xenon is being continuously purified by two hot metal getters manufactured by $S E A S$ [18], through two separate circulation loops with stable flow rates of about 80 and 30 standard-liter-per-minute (slpm), respectively. Purified xenon gas is driven by diaphragm pumps into two heat exchangers located close to the detector. Inside the heat exchangers, purified gas is cooled and liquefied by liquid xenon extracted from the detector.

The sensitive target is a cylindrical dual phase xenon TPC confined by 24 highly reflective polytetrafluoroethylene (PTFE) wall panels, with an opposite-panel distance of $1185 \mathrm{~mm}$ (room temperature). The electrical fields in the TPC are defined by, from the bottom to the top, a cathode grid, a gate mesh, and an anode mesh, with a separation of 1185 and $10 \mathrm{~mm}$ in between. The liquid level is set in between the gate and anode by the top opening of the overflow tube, which is adjustable externally via a motion feedthrough. Under an electrical field, the average gas gap is $3.5 \mathrm{~mm}$ from the anode, and the relative distortion between the gate and anode, primarily due to electrostatic attraction, is less than $0.4 \mathrm{~mm}$. A total of 169 and 199 of Hamamatsu R11410-23 three-inch photomultipliers (PMTs) are located at the top and bottom of the TPC, respectively, with grounded screening meshes $6 \mathrm{~mm}$ away from the PMT surfaces. During the operation, nine R11410-23 PMTs were turned off due to connection or base problems, and four PMTs were turned off due to excessive noise. The average dark rate for the remaining
PMTs is about $100 \mathrm{~Hz}$ per channel. The prompt scintillation photons $(S 1)$ and delayed electroluminescence photons $(S 2$, proportional to the number of ionized electrons extracted into the gaseous region) are measured by the top and bottom PMT arrays. This allows, for a given event, a three-dimensional vertex reconstruction to a subcentimeter precision. The outside wall of the field cage is about $70 \mathrm{~mm}$ from the inner cryostat, to leave enough space for the cathode feedthrough. Two rings of Hamamatsu R8520 one-inch PMTs (105 in total) are instrumented in this gap facing upward and downward, respectively, serving as the background veto. The PMT gains are calibrated, once per week, by four external blue light-emitting diodes with photons transmitted into the detector via optical fibers. The average gains of the PMTs are $5.5 \times 10^{6}$ for R11410-23 and $2.3 \times 10^{6}$ for R8520. The PMT pulses are amplified by low-noise linear amplifiers with a gain of 1.5 and 5 for R11410-23 and R8520 PMTs, respectively, and then digitized by the CAEN V1725B digitizer with $0.122 \mathrm{mV}$ per analog-to-digital-convertor (ADC) bit and a sampling rate of $2.5 \times 10^{8}$ samples per second [19]. The digitizers are operated under the self-trigger mode, so if any pulse is above a predefined threshold corresponding to about $1 / 3$ of a photoelectron (PE), the entire waveform is read out $[20,21]$. The readout efficiency for a single PE is measured for each channel, with an average value of $96 \%$. The data are read out through optical fibers and directly stored onto the disk. Physical events are reconstructed via off-line software [22].

An off-line krypton distillation was carried out on all 5.6 tonne of xenon using a newly constructed distillation tower at CJPL [23]. The detector was then filled, and, after basic functionality checks, the water shield was filled with ultrapure water which has electrical resistivity of about $18 \mathrm{MOhms} \cdot \mathrm{cm}$ and concentration of uranium and thorium less than 0.1 ppt. The commissioning run of PandaX-4T commenced on November 28, 2020 and ended on April 16, 2021 , including 95.0 calendar days of stable data taking. In this period, the diaphragms of the circulation pumps were worn out two times, each time causing degradation in electron drifting, but with no trace of radioactive impurity introduced. The cathode and gate voltages were set at several different values to avoid excessive discharges, separating the data into several sets. The liquid level was adjusted between sets 2 and 3. During set 4, the online krypton distillation was kept on with a flow rate of $10 \mathrm{slpm}$. The detailed run configurations can be found in Table I.

The data processing follows a similar procedure as in the previous PandaX analysis [9]. Hits with amplitudes larger than $20 \mathrm{ADC}(\sim 2.44 \mathrm{mV})$ are identified from the waveform of individual channels. Signals are defined as clusters of hits with a tail-to-head gap no greater than 15 samples (60 ns), corresponding to an approximate $104 \mathrm{~ns}$ peakto-peak separation between hits, and a coincidence 
TABLE I. Basic detector configurations of the commissioning datasets, with $\left\langle\tau_{e}\right\rangle, d t_{\max }$, PDE, EEE, and $\mathrm{SEG}_{b}$ representing the average electron lifetime, maximum drift time, photon detection efficiency, electron extraction efficiency, and single-electron gain from the bottom PMT array (with a total-to-bottom ratio of 4.2), respectively.

\begin{tabular}{lccccc}
\hline \hline Set & 1 & 2 & 3 & 4 & 5 \\
\hline Duration (days) & 1.95 & 13.25 & 5.53 & 35.58 & 36.51 \\
$\left\langle\tau_{e}\right\rangle(\mu \mathrm{s})$ & 800.4 & 939.2 & 833.6 & 1121.5 & 1288.2 \\
$d t_{\max }(\mu \mathrm{s})$ & 800 & 810 & 817 & 841 & 841 \\
$V_{\text {cathode }}(-\mathrm{kV})$ & 20 & 18.6 & 18 & 16 & 16 \\
$V_{\text {gate }}(-\mathrm{kV})$ & 4.9 & 4.9 & 5 & 5 & 5 \\
PDE $(\%)$ & $9.0 \pm 0.2$ & & $9.0 \pm 0.2$ & \\
EEE $(\%)$ & $90.2 \pm 5.4$ & & $92.6 \pm 5.4$ & \\
$\mathrm{SEG}_{b}(\mathrm{PE} / e)$ & $3.8 \pm 0.1$ & & $4.6 \pm 0.1$ & \\
\hline \hline
\end{tabular}

requirement that at least two different PMTs receive hits. The inefficiency of such a clustering gap requirement is validated to be negligible using data-driven approaches. Other unphysical noises are identified by anomalous shapes or charge distribution pattern. Signals are then classified into $S 1$-like and $S 2$-like according to number of hits, the charge ratio between the top and bottom arrays, and width of the waveform enclosing 10\%-90\% cumulative charge $\left(w_{\text {cum }}\right)$. S2-like signals are reclustered by taking into account the diffusion effect during the drift. The inefficiency of tagging is verified to be negligible. S1-like and S2-like signals within a window of $1 \mathrm{~ms}$ are further combined into an event.

Three classes of data quality cuts are developed based on the calibration data (see later) and on our practice in previous generations of PandaX, to remove noise and unphysical events. (i) A set of "waveform cleanliness" cuts is applied to avoid having too much noise or too many single-electron $S 2$ s within an event. (ii) For $S 1$ s, to avoid confusions with single-electron $S 2$ s, the number of peaks in a summed waveform is required to be no more than four, and the top-bottom charge ratio should be consistent with the location of the interaction. Another cut is applied on the charge distribution to suppress abnormal charge caused by PMT after pulsing. (iii) For $S 2 \mathrm{~s}$, cuts are applied to their waveform shapes, top-bottom charge ratio, the root-meansquare (rms) of the charge distribution on the top PMTs, and the quality of horizontal position reconstruction. An $S 2$-dependent cut on drift time vs $w_{\text {cum }}$ is made, which is important to suppress the accidental background. Unless otherwise specified, a good event should have only one pair of $S 1$ and $S 2$.

The position of an event is reconstructed using the charge pattern on the top PMT array (horizontal) and the drift time (vertical) assuming a constant drift velocity. Two independent horizontal reconstruction methods have been developed, the template matching method and the photon acceptance function method [24]. Only the first half of the charge in $S 2$ is used in the reconstruction, leading to a better position resolution in comparison to that using the total charge. The position uncertainty in the vertical direction is conservatively estimated to be $3 \mathrm{~mm}$, based on the width of the $S 2$. In the horizontal plane, the reconstruction uncertainty depends on the charge of $S 2$ and is estimated to be 8.2 (100 PE) and $3.0 \mathrm{~mm}(1000 \mathrm{PE})$ based on the comparison between the two methods, cross-checked with the sigma of the radial distribution of the surface events from the PTFE wall.

The uniformity of detector responses is calibrated using internal diffusive sources. To avoid PMT saturations, the bottom-only $S 2\left(S 2_{b}\right)$ is used. The vertical uniformity of $S 2_{b}$, characterized by the electron lifetime $\tau_{e}$, is calibrated using the $164 \mathrm{keV}$ deexcitation peak from ${ }^{131} \mathrm{~m} \mathrm{Xe}$ (produced by neutron irradiation), which is found consistent with that obtained from radon alpha peaks. The average $\tau_{e}$ in each run set is summarized in Table I. The threedimensional uniformity of $S 1$ and the horizontal uniformity of $S 2_{b}$ are calibrated by injecting ${ }^{83 \mathrm{~m}} \mathrm{Kr}(41.5 \mathrm{keV})$ into the detector via one of the circulation loops [25]. This calibration was carried out twice, at the beginning and completion of the commissioning data taking, with about 100000 events collected. In the fiducial volume (FV, defined later), the rms variation in $S 1$ and horizontal $S 2_{b}$ responses is $19 \%$ and $15 \%$, respectively.

The electron-equivalent energy $E$ of a given event can be reconstructed as [26]

$$
E=13.7 \mathrm{eV} \times\left(\frac{S 1}{\mathrm{PDE}}+\frac{S 2_{b}}{\mathrm{EEE} \times \mathrm{SEG}_{b}}\right),
$$

in which PDE, EEE, and $\mathrm{SEG}_{b}$ are the photon detection efficiency for $S 1$, electron extraction efficiency, and the single-electron gain using $S 2_{b}$, respectively, and $13.7 \mathrm{eV}$ is the work function in LXe. The value of $\mathrm{SEG}_{b}$ is measured by selecting the smallest $S 2_{b}$, with an enlarged 50-sample clustering gap requirement. The rms of the $\mathrm{SEG}_{b}$ in the $\mathrm{FV}$ is $8 \%$. The PDE and EEE are fitted according to Eq. (1) using the following electron recoil (ER) peaks:

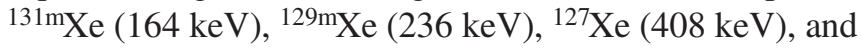
${ }^{83 \mathrm{~m}} \mathrm{Kr}(41.5 \mathrm{keV})$. The PDE, EEE, and $\mathrm{SEG}_{b}$ in different datasets are summarized in Table I.

The low-energy calibration is carried out after set 5 . The ER response is calibrated by injecting ${ }^{220} \mathrm{Rn}$ into the detector. The ${ }^{220} \mathrm{Rn}$ is produced by a foil ${ }^{228} \mathrm{Th}$ source with an expected ${ }^{220} \mathrm{Rn}$ emanation rate of $240 \mathrm{~Bq}$. The ${ }^{220} \mathrm{Rn}$ rate observed in the detector is about 1.7 Bq. In total, 1393 lowenergy single-scatter ER events are collected within an $S 1$ range from 2 to $135 \mathrm{PE}$ in the FV. The distribution of the ER calibration events in $\log _{10}\left(n_{e} / S 1\right)$ vs $S 1$ can be found in Fig. 1 , where $n_{e}$ is defined as $S 2_{b} /\left(\mathrm{EEE} \times \mathrm{SEG}_{b}\right)$.

The NR response is calibrated with two different neutron sources. The ${ }^{241} \mathrm{Am}-\mathrm{Be}$ source is deployed through three external horizontal tubes outside the inner cryostat at three 


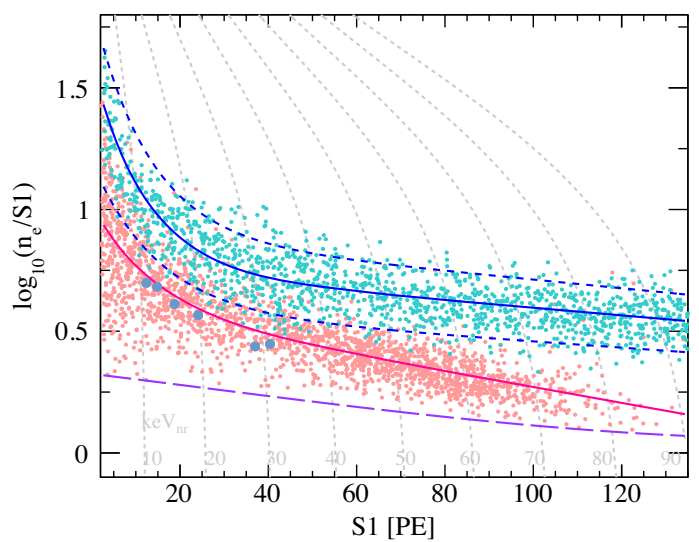

FIG. 1. The distributions of ${ }^{220} \mathrm{Rn}$ (cyan line) and D-D (magenta line) calibration events in $\log _{10}\left(n_{e} / S 1\right)$ vs $S 1$. The solid blue and red lines represent the fitted ER and NR medians, respectively, and the dashed blue lines are the corresponding $95 \%$ quantiles of ER events. The D-D neutrons are selected within 120-520 $\mu$ s in drift time to avoid the so-called neutron-X events with partial energy deposition below the cathode. The six ER events from ${ }^{220} \mathrm{Rn}$ calibration data located below the NR median line are highlighted. The dashed violet line represents the $99.5 \% \mathrm{NR}$ acceptance cut. The nuclear recoil energy in $\mathrm{keV}_{\mathrm{nr}}$ is indicated with the gray dashed lines.

different heights of the TPC. The deuteron-deuteron (D-D) neutrons are collimated horizontally via a beam pipe intruded into the water tank pointing to the center of the TPC. The distribution of the single-scatter D-D NR events is overlaid in Fig. 1.

Since the data selection is made in $S 1$ and $S 2$, the total efficiency as a function of energy includes two major components, the signal reconstruction and detection efficiency, and the data quality cut efficiency. The signal reconstruction and detection efficiency is the ability for the readout and off-line software to correctly identify a true $S 1 / S 2$, including the readout threshold (so-called BLS nonlinearity in Ref. [9]), signal clustering efficiency (15-sample), and the $S 1 / S 2$ classification efficiency, all determined using data-driven methods. It also takes into account the event loss due to the requirements on number of hits $(\geq 2)$ and selection ranges of $S 1$ and $S 2$, determined using the signal model simulation. The efficiency of the data quality cuts is determined using the calibration data by taking the calibration events within the 5\%-95\% quantiles in Fig. 1 and calculating the ratio of number of events with all cuts applied and that with all-but-this cut for the three classes of cuts described above. The efficiencies separately determined from ${ }^{220} \mathrm{Rn}, \mathrm{AmBe}$, and D-D calibration data are all consistent. The total efficiency vs nuclear recoil energy is shown in Fig. 2.

A standard unbinned likelihood function is defined to perform a simultaneous fit of ER and NR response models based on the calibration data $\left({ }^{220} \mathrm{Rn},{ }^{241} \mathrm{Am}-\mathrm{Be}\right.$, and D-D) in $\left(S 1, S 2_{b}\right)$, with fast detector simulation including the

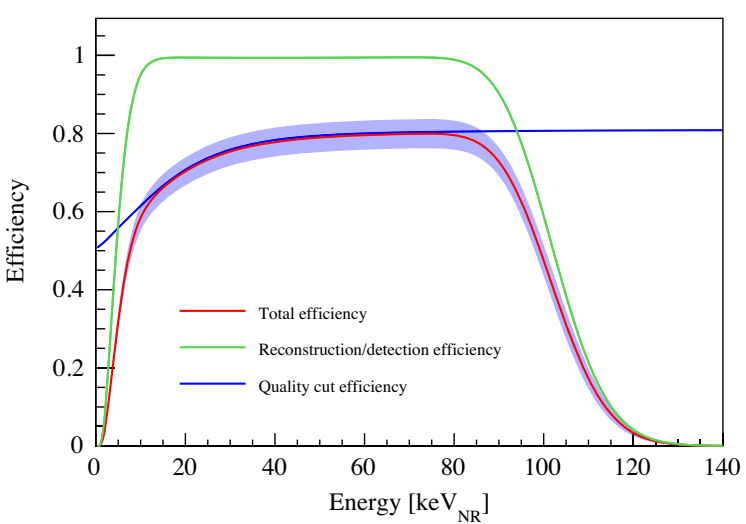

FIG. 2. The reconstruction and detection efficiency (green line), data quality cut efficiency (blue line), and the total efficiency (red, with the shaded band representing the uncertainty) as a function of the nuclear recoil energy.

following effects: photon detection, electron drifting, diffusion, extraction and amplification, nonuniformities of $S 1$ and $S 2_{b}$, and detection efficiencies [27,28]. The ER and NR response models follow the standard NEST 2.0 construction [29,30], with the light yield, charge yield, and recombination parameters fitted. The likelihood function is minimized via Markov chain Monte Carlo [31], with fast detector simulation $[27,28]$ boosted on GPUs. The best fit parameters are consistent with their nominal values in NEST 2.0. The NR models obtained with AmBe or D-D data only are also in good agreement. Binned-log-likelihood goodness-of-fit tests were made, with a resulting $p$ value of 0.38 and 0.78 for the ER and NR calibration data, respectively. For sets $1-3$, due to small differences in drift field in comparison to sets 4 and 5, the response model of sets 1-3 is extrapolated from that of sets 4 and 5 in accordance with the field dependence of light and charge yields in NEST $2.0[29,30]$, from which a less-than-1\% difference in the light yield is predicted. The same value of PDE is used for sets 1-3, but $\mathrm{SEG}_{b}$ and EEE (scaled from in situ ER peaks) are separately determined.

Aside from detector or data acquisition downtime, to eliminate stray electrons due to a previous energetic interaction, candidate events have to be separated by $22 \mathrm{~ms}$ from a previous event so that the contamination from leftover pulses can be neglected. This inserts a wellcontrolled dead time of approximately $7.3 \%$. Data with abnormal isolated $S 1$ rate, indicating excessive discharges from the electrodes and PMTs, are also removed from the analysis, reducing the live time by about $2.3 \%$. The resulting live time is 86.0 days. The dark matter candidates are selected using the following cut criteria. The ranges of $S 1$ and $S 2$ are [2, 135] and [80, 20 000] PE, respectively. The veto PMT is required to see no coincidental photons during an $S 1$. The events are also required to be above the 99.5\% NR quantile (see Fig. 1). The FV mass of about 2.67 tonne (with an uncertainty of $1.7 \%$ ), indicated 
TABLE II. Expected background contributions to dark matter candidates for individual datasets. The "flat ER (data)" refers to a combination of radon, krypton, detector material background, solar neutrino, and ${ }^{136} \mathrm{Xe}$. For better statistical uncertainty, it is independently derived set by set from the data within the energy range from 18 to $30 \mathrm{keV}$, which is then used in the final likelihood fit. The tritium values are obtained from unconstrained fit. The neutron, ${ }^{8} \mathrm{~B}$, surface, and accidental background are assumed to be constant throughout the run. The background-only best fit values and uncertainties are also shown, where the central values are used to generate pseudo datasets for the calculation of sensitivity.

\begin{tabular}{|c|c|c|c|c|c|c|c|c|}
\hline & Set 1 & Set 2 & Set 3 & Set 4 & Set 5 & Total & Below NR median & Best fit \\
\hline $\mathrm{Rn}$ & $6.9 \pm 3.8$ & $42.8 \pm 23.5$ & $22.7 \pm 12.5$ & $162.0 \pm 88.9$ & $112.1 \pm 61.5$ & $346.5 \pm 190.2$ & $1.42 \pm 0.78$ & . \\
\hline $\mathrm{Kr}$ & $1.1 \pm 0.7$ & $7.7 \pm 4.9$ & $3.2 \pm 2.1$ & $20.4 \pm 13.1$ & $20.9 \pm 13.4$ & $53.3 \pm 34.2$ & $0.21 \pm 0.13$ & \\
\hline Material & $0.8 \pm 0.1$ & $5.7 \pm 0.7$ & $2.4 \pm 0.4$ & $15.2 \pm 1.9$ & $15.6 \pm 1.9$ & $39.7 \pm 5.0$ & $0.16 \pm 0.02$ & $\ldots$ \\
\hline Solar $\nu$ & $0.8 \pm 0.2$ & $5.4 \pm 1.1$ & $2.3 \pm 0.5$ & $14.3 \pm 2.9$ & $14.6 \pm 2.9$ & $37.4 \pm 7.5$ & $0.16 \pm 0.03$ & \\
\hline${ }^{136} \mathrm{Xe}$ & $0.7 \pm 0.1$ & $4.6 \pm 0.9$ & $1.9 \pm 0.4$ & $11.8 \pm 2.4$ & $12.1 \pm 2.4$ & $31.1 \pm 6.2$ & $0.05 \pm 0.01$ & $\cdots$ \\
\hline Flat ER (data) & $4.0 \pm 2.9$ & $54.5 \pm 10.5$ & $12.2 \pm 4.9$ & $240.5 \pm 21.8$ & $180.9 \pm 18.9$ & $492.1 \pm 31.2$ & $2.06 \pm 0.14$ & $509.6 \pm 22.8$ \\
\hline $\mathrm{CH}_{3} \mathrm{~T}$ & $17 \pm 5$ & $88 \pm 11$ & $21 \pm 6$ & $258 \pm 24$ & $148 \pm 17$ & $532 \pm 32$ & $5.1 \pm 0.3$ & $532 \pm 32$ \\
\hline${ }^{127} \mathrm{Xe}$ & $0.19 \pm 0.04$ & $1.08 \pm 0.25$ & $0.96 \pm 0.22$ & $3.99 \pm 0.92$ & $1.91 \pm 0.44$ & $8.13 \pm 1.07$ & $0.12 \pm 0.02$ & $8.41 \pm 2.08$ \\
\hline Neutron & $0.02 \pm 0.01$ & $0.15 \pm 0.08$ & $0.07 \pm 0.03$ & $0.45 \pm 0.22$ & $0.46 \pm 0.23$ & $1.15 \pm 0.57$ & $0.69 \pm 0.35$ & $0.82 \pm 0.41$ \\
\hline${ }^{8} \mathrm{~B}$ & $0.01 \pm 0.01$ & $0.05 \pm 0.03$ & $0.03 \pm 0.02$ & $0.26 \pm 0.13$ & $0.29 \pm 0.15$ & $0.64 \pm 0.32$ & $0.62 \pm 0.31$ & $0.61 \pm 0.17$ \\
\hline Surface & $0.01 \pm 0.01$ & $0.07 \pm 0.02$ & $0.03 \pm 0.01$ & $0.18 \pm 0.05$ & $0.18 \pm 0.05$ & $0.47 \pm 0.13$ & $0.42 \pm 0.12$ & $0.44 \pm 0.11$ \\
\hline Accidental & $0.04 \pm 0.01$ & $0.32 \pm 0.05$ & $0.03 \pm 0.01$ & $0.99 \pm 0.18$ & $1.05 \pm 0.21$ & $2.43 \pm 0.47$ & $0.80 \pm 0.15$ & $2.31 \pm 0.45$ \\
\hline Sum & $21 \pm 6$ & $144 \pm 15$ & $34 \pm 8$ & $504 \pm 32$ & $333 \pm 25$ & $1037 \pm 45$ & $9.8 \pm 0.6$ & $1054 \pm 39$ \\
\hline Data & 21 & 148 & 34 & 496 & 359 & 1058 & 6 & \\
\hline
\end{tabular}

in Fig. 3(b), is determined based on the expected background distributions to optimize the sensitivity, with the material background from simulation, the internal contamination from data-driven estimate, and neutron background from a combined data-driven (rate) and simulation (vertex distribution) estimate.

The following major background components are considered in the dark matter analysis, with their rates summarized in Table II.

The detector materials have been assayed by the highpurity germanium detector, and the background due to material radioactivity is dominated by the PMTs and the stainless steel vessels [10]. The energy and position distribution of the high-energy gammas in the data are consistent with expectations from simulation, and the integrated rates above $1 \mathrm{MeV}$ agree within 14\%. The expected contribution to background in the dark matter window is $40 \pm 5$ events.

The radon background rate is measured in situ using alpha events. The decay of ${ }^{222} \mathrm{Rn}$ is $4.2 \pm 0.1 \mu \mathrm{Bq} / \mathrm{kg}$ during sets $1,2,3$, and 5 and $5.9 \pm 0.1 \mu \mathrm{Bq} / \mathrm{kg}$ during set 4 (increased due to radon emanation from the distillation tower during the online krypton distillation), and that from ${ }^{220} \mathrm{Rn}$ is $0.07 \pm 0.01 \mu \mathrm{Bq} / \mathrm{kg}$. The expected low-energy radon background is dominated by ${ }^{214} \mathrm{~Pb} \beta$ 's (decay lifetime $\sim 39 \mathrm{~min}$ ), which is not equally populated as their ancestors in the TPC as positive ions tend to drift toward and attach to the cathode [32]. Its contribution is determined by taking the difference of the low-energy rates between sets 4 and 5 . The overall contribution to the dark matter background is $347 \pm 190$ events.
The ${ }^{85} \mathrm{Kr} \beta$-decay background is estimated based on a correlated emission of $\beta-\gamma$ through the metastable state ${ }^{85 \mathrm{~m}} \mathrm{Rb}(514 \mathrm{keV}, 0.43 \%)$. Assuming a $2 \times 10^{-11}$ isotopic concentration of ${ }^{85} \mathrm{Kr}$ [33], a $\mathrm{Kr} / \mathrm{Xe}$ ratio of $0.33 \pm$ $0.21 \mathrm{ppt}$ is found. The expected background is $53 \pm 34$ events.

ER background due to solar neutrinos is estimated assuming the standard solar model, three-neutrino-flavor oscillation, and the standard model anomalous magnetic moment [34]. ${ }^{136} \mathrm{Xe}$ two-neutrino beta decay is computed using the lifetime from Ref. [35]. The backgrounds from radon, krypton, detector materials, solar neutrino, and ${ }^{136} \mathrm{Xe}$ are combined into a "flat ER (data)" background in Table II, independently derived from data within the energy range from 18 to $30 \mathrm{keV}$, and applied in the final dark matter fit.

Some number of tritium events are identified in the data. The origin is likely due to some leftover tritium from PandaX-II end-of-run calibration [36]. The event rate is allowed to float independently for each set, with a total fitted $532 \pm 32$ events in the FV and an average concentration of $5 \times 10^{-24} \mathrm{~mol} / \mathrm{mol}$ in xenon. The temporal variation in the data (Table II), particularly in between sets 4 and 5, indicates that gas circulation through hot getters may slowly reduce its concentration.

Some cosmogenically activated ${ }^{127} \mathrm{Xe}$ is also identified in the data, which decays through electron captures. The background due to $L$-shell captures $(5.2 \mathrm{keV})$ is estimated based on the measured $K$-shell captures in the FV $(33.2 \mathrm{keV})$ and their expected ratio $(1: 6)$ to be $8 \pm 1$ events. Its decay (mean lifetime 52.5 days) is considered set by set in the final fit. 
The long-lived progenies of radon attached onto the PTFE surface also contribute to the background, for example, through ${ }^{210} \mathrm{~Pb} \beta$ decays. These events have a much suppressed $S 2$ signal, likely due to the loss of electrons on the PTFE surface during the drift. The radial distributions of these events in different $S 2_{b}$ bins are obtained using ${ }^{210} \mathrm{Po}$ surface events, tagged by $\mathrm{S} 1 \mathrm{~s}$ peaking around $30000 \mathrm{PE}(5.3 \mathrm{MeV})$. The expected distribution in $S 1$ and $S 2_{b}$ and the rate normalization is obtained using events reconstructed outside the PTFE wall but otherwise within the dark matter selection. The residual background in the FV is $0.5 \pm 0.1$ events.

The neutron background in the data is estimated using three methods. The first method is described in Ref. [10], but with updated radioactivities, selection efficiency, and veto efficiency. The second method uses the single-scatter to multiscatter ratio of NR events. The third method follows the procedure in Ref. [37], with a predicted ratio between the single-scatter NR and high-energy neutron capture gammas. The residual neutron background in the dark matter data is $1.2 \pm 0.6$ events.

${ }^{8} \mathrm{~B}$ neutrinos from the Sun can make coherent neutrinonucleus scattering with xenon nucleus [38]. This background is estimated to be $0.6 \pm 0.3$ events.

The accidental background due to randomly paired $S 1$ and $S 2$ is studied by first identifying isolated $S 1$ and $S 2$ events, with a rate of $9.5(S 1)$ and $0.0045 \mathrm{~Hz}(S 2)$ and a standard deviation of $10.5 \%(S 1)$ and $12.7 \%(S 2)$, derived based on rates at different data-taking periods. The isolated $S 1$ s and $S 2$ s are randomly assembled in time, with selection cuts applied afterward. The remaining background in the dark matter sample is $2.4 \pm 0.5$ events, consistent with that obtained by selecting $S 1$ s and $S 2$ s from the data with a time separation beyond the maximum drift time.

Within the FV and dark matter selection window, 1058 final candidate events are identified. To take into account the difference in EEE and $\mathrm{SEG}_{b}$ between sets 1 and 2 and $3-5$, we define $n_{e}=S 2_{b} / \mathrm{EEE} / \mathrm{SEG}_{b}$; thereby, the distribution of all events in $\log _{10}\left(n_{e} / S 1\right)$ vs $S 1$ is shown in Fig. 3(a). Six events are identified below the NR median curve. Candidates are uniformly distributed in the FV, with position distributions in $z$ vs $r^{2}$ and $y$ vs $x$ displayed in Figs. 3(b) and 3(c).

Dark matter signals are searched in our data using a profile likelihood ratio (PLR) approach with a double-sided statistic construction [39]. At each dark matter mass $m_{\chi}$ and its spin-independent (SI) elastic cross section with the nucleon $\sigma_{\chi, n}$, the NR rate and spectrum of the signal is computed using the recipe in Ref. [39]. The probability density functions (PDFs) of the background and dark matter signals are both produced in $S 1$ and $S 2_{b}$ using the aforementioned response models. A standard unbinned likelihood function is constructed [9], with Gaussian penalty terms defined according to the uncertainty of the (a) $\log _{10}\left(n_{e} / S 1\right)$ vs. $S 1$

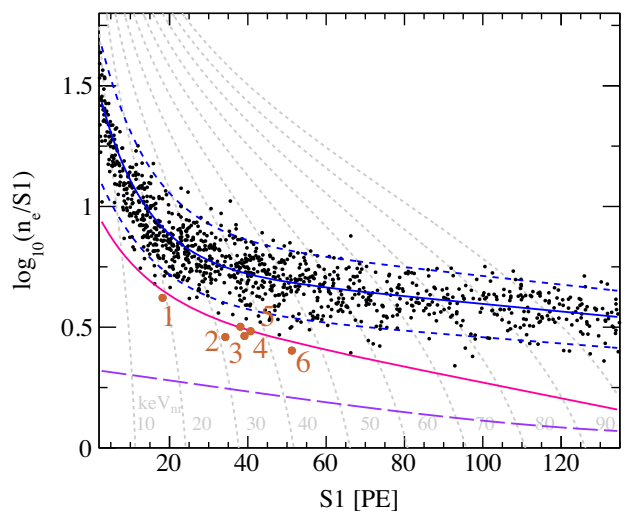

(b) $z$ vs. $r^{2}$

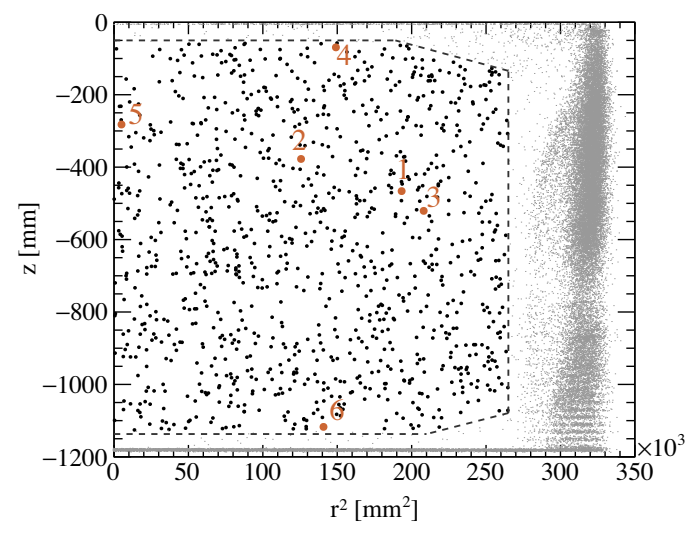

(c) $y$ vs. $x$

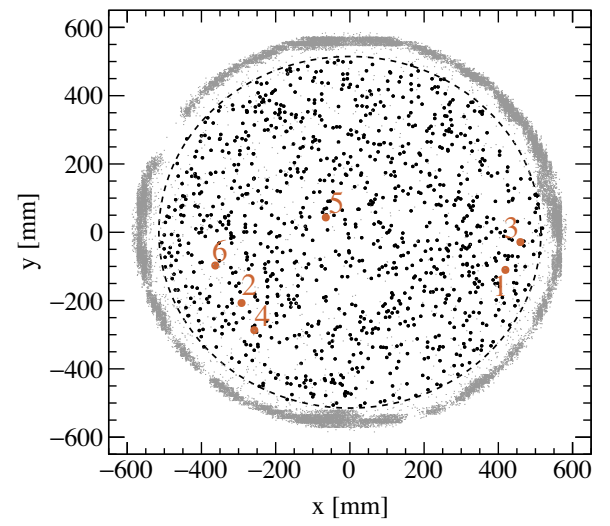

FIG. 3. Distributions of the final dark matter candidates in $\log _{10}\left(n_{e} / S 1\right)$ vs $S 1$ (a), $z$ vs $r^{2}$ (b), and $y$ vs $x$ (c). In (a), the solid blue and red lines are the ER and NR medians, respectively, and the dashed blue lines are the corresponding $95 \%$ quantiles of ER events. The dashed violet line represents the $99.5 \%$ NR acceptance cut. The nuclear recoil energy in $\mathrm{keV}_{\mathrm{nr}}$ is indicated with the gray dashed lines. The six ER events located below the NR median line are highlighted in brown, with No. 1 from set 3, No. 2, No. 4, and No. 6 from set 4, No. 3 from set 2, and No. 5 from set 5 . In (b) and (c), the dashed lines are projections of the FV, and black (light gray) dots represent events inside (outside). 


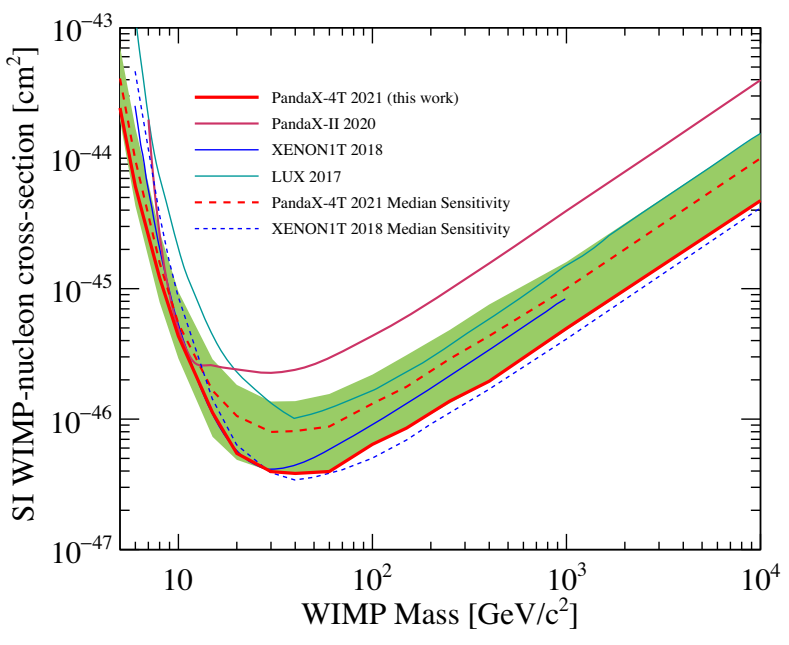

FIG. 4. The $90 \%$ C.L. upper limit from this work (unblind analysis) vs $m_{\chi}$ for the SI WIMP-nucleon elastic cross section, overlaid with that from the full datasets of LUX 2017 [6], XENON1T 2018 [8], and PandaX-II 2020 [9], obtained using blinded or salted analyses. The green band represents the $\pm 1 \sigma$ sensitivity band. The black and red dashed curves represent the median sensitivities of XENON1T and PandaX-4T, respectively. Results from XENON1T, LUX, or PandaX-II partial datasets are not included in the figure.

parameters in the response models and rates of individual background (Table II). For our background-only fit, the goodness-of-fit $p$ value is 0.71 . PLR scans are made on grids of $\left(m_{\chi}, \sigma_{\chi, n}\right)$. No clear excess over background is observed. In Fig. 4, the 90\% C.L. upper limit of SI cross section from our data is shown, together with $\pm 1 \sigma$ sensitivity band obtained from background-only pseudo data, as well as limits from previous experiments $[6,8,9]$. Our median sensitivity has improved from the PandaX-II final analysis [9] by 2.6 times at $m_{\chi}$ of $40 \mathrm{GeV} / c^{2}$. Our limit is within the $\pm 1 \sigma$ sensitivity band for $m_{\chi}$ below $25 \mathrm{GeV} / c^{2}$ and goes slightly beyond $-1 \sigma$ until about $250 \mathrm{GeV} / c^{2}$, indicating a downward fluctuation of the background. The limit is, therefore, conservatively power constrained to $-1 \sigma$ [40]. In comparison to XENON1T's final result, for $m_{\chi}$ below $20 \mathrm{GeV} / c^{2}$, our median sensitivity and exclusion limit are both stronger, which is primarily driven by our higher efficiency below $4 \mathrm{keV}_{\mathrm{nr}}$, attributed to the two-hit coincidence requirement. On the other hand, our median sensitivity is weaker than XENON1T for $m_{\chi}$ beyond $20 \mathrm{GeV} / c^{2}$, approaching a factor of 2.5 times or so for high-mass dark matter (DM). This is expected from the exposure (0.63 vs 1 tonne-year), efficiency difference at high recoil energy, and our higher background level due to tritium contamination. More information can be found in the supplemental material [41]. Our new limit represents the most stringent constraint to DM-nucleon SI interactions, with the lowest excluded cross section value of $3.8 \times 10^{-47} \mathrm{~cm}^{2}$ at $m_{\chi}$ of $40 \mathrm{GeV} / c^{2}$.

In summary, we report the dark matter search results using the commissioning data from PandaX-4T, with a live exposure of 0.63 tonne year. No dark matter candidates are identified above expected background. The strongest upper limit to date is set on the dark matter-nucleon spinindependent interactions, with the lowest excluded value of $3.8 \times 10^{-47} \mathrm{~cm}^{2}$ at $40 \mathrm{GeV} / c^{2}$. PandaX-4T is undertaking a tritium removal campaign, after which normal physics data taking will start. The dark matter search sensitivity is expected to improve by another order of magnitude with a 6-tonne $\cdot$ year exposure.

This project is supported in part by a grant from the Ministry of Science and Technology of China (No. 2016YFA0400301), grants from National Science Foundation of China (No. 12090060, No. 12005131, No. 11905128, No. 11925502, and No. 11775141), and by Office of Science and Technology, Shanghai Municipal Government (Grant No. 18JC1410200). We thank supports from Double First Class Plan of the Shanghai Jiao Tong University. We also thank the sponsorship from the Chinese Academy of Sciences Center for Excellence in Particle Physics (CCEPP), Hongwen Foundation in Hong Kong, and Tencent Foundation in China. Finally, we thank the CJPL administration and the Yalong River Hydropower Development Company Ltd. for indispensable logistical support and other help.

*Corresponding author. qinglin@ustc.edu.cn Spokesperson. jianglai.liu@sjtu.edu.cn Corresponding author. nzhou@sjtu.edu.cn ${ }^{\S}$ Corresponding author. zhou_xp@buaa.edu.cn

[1] G. Bertone, D. Hooper, and J. Silk, Phys. Rep. 405, 279 (2005).

[2] J. Liu, X. Chen, and X. Ji, Nat. Phys. 13, 212 (2017).

[3] J. Conrad and O. Reimer, Nat. Phys. 13, 224 (2017).

[4] O. Buchmueller, C. Doglioni, and L.-T. Wang, Nat. Phys. 13, 217 (2017).

[5] J. Billard et al., arXiv:2104.07634.

[6] D. Akerib et al. (LUX Collaboration), Phys. Rev. Lett. 118, 021303 (2017).

[7] X. Cui et al. (PandaX Collaboration), Phys. Rev. Lett. 119, 181302 (2017).

[8] E. Aprile et al. (XENON Collaboration), Phys. Rev. Lett. 121, 111302 (2018).

[9] Q. Wang et al. (PandaX Collaboration), Chin. Phys. C 44, 125001 (2020).

[10] H. Zhang et al. (PandaX Collaboration), Sci. China Phys. Mech. Astron. 62, 31011 (2019).

[11] B. J. Mount et al., arXiv:1703.09144. 
[12] E. Aprile et al. (XENON Collaboration), J. Cosmol. Astropart. Phys. 11 (2020) 031.

[13] K. J. Kang, J. P. Cheng, Y. H. Chen, Y. J. Li, M. B. Shen, S. Y. Wu, and Q. Yue, J. Phys. Conf. Ser. 203, 012028 (2010).

[14] J. Li, X. Ji, W. Haxton, and J. S. Y. Wang, Phys. Procedia 61, 576 (2015).

[15] T. Zhang, C. Fu, X. Ji, J. Liu, X. Liu, X. Wang, C. Yao, and X. Yuan, J. Instrum. 11, T09004 (2016).

[16] X. Cao et al., Sci. China Phys. Mech. Astron. 57, 1476 (2014).

[17] L. Zhao, X. Cui, W. Ma, Y. Fan, K. Giboni, T. Zhang, J. Liu, and X. Ji, J. Instrum. 16, T06007 (2021).

[18] SAES Pure Gas, https://www.entegris.com/shop/en/USD/ Products/Gas-Filtration-and-Purification/Gas-Purifiers/c/ gaspurifiers, 2021 (accessed November 30, 2021).

[19] See https://www.caen.it/products/v1725/.

[20] Q. Zheng, Y. Huang, D. Huang, J. Liu, X. Ren, A. Wang, M. Wang, J. Yang, B. Yan, and Y. Yang, J. Instrum. 15, T12006 (2020).

[21] J. Yang et al., arXiv:2108.03433.

[22] X. Chen et al. (to be published).

[23] X. Cui et al., J. Instrum. 16, P07046 (2021).

[24] D. Zhang et al., arXiv:2106.08380.

[25] D. Zhang et al., arXiv:2102.02490.

[26] M. Szydagis, N. Barry, K. Kazkaz, J. Mock, D. Stolp, M. Sweany, M. Tripathi, S. Uvarov, N. Walsh, and M. Woods, J. Instrum. 6, P10002 (2011).

[27] B. Yan et al. (PandaX Collaboration), Chin. Phys. C 45, 075001 (2021).
[28] E. Aprile et al. (XENON Collaboration), Phys. Rev. D 99, 112009 (2019).

[29] M. Szydagis et al., Noble element simulation technique v2.0, 2018, https://zenodo.org/record/1314669.

[30] M. Szydagis et al., Instruments 5, 13 (2021).

[31] D. Foreman-Mackey, D. W. Hogg, D. Lang, and J. Goodman, Publ. Astron. Soc. Pac. 125, 306 (2013).

[32] W. Ma et al., J. Instrum. 15, P12038 (2020).

[33] P. Collon, W. Kutschera, and Z.-T. Lu, Annu. Rev. Nucl. Part. Sci. 54, 39 (2004).

[34] J. Billard, E. Figueroa-Feliciano, and L. Strigari, Phys. Rev. D 89, 023524 (2014).

[35] J. B. Albert et al. (EXO-200 Collaboration), Phys. Rev. C 89, 015502 (2014).

[36] X. Zhou et al. (PandaX Collaboration), Chin. Phys. Lett. 38, 011301 (2021).

[37] Q. Wang et al. (PandaX Collaboration), Sci. China Phys. Mech. Astron. 63, 231011 (2020).

[38] F. Ruppin, J. Billard, E. Figueroa-Feliciano, and L. Strigari, Phys. Rev. D 90, 083510 (2014).

[39] D. Baxter et al., Eur. Phys. J. C 81, 907 (2021).

[40] G. Cowan, K. Cranmer, E. Gross, and O. Vitells, arXiv: 1105.3166.

[41] See Supplemental Material at http://link.aps.org/ supplemental/10.1103/PhysRevLett.127.261802 for the detector response measurement, total signal efficiency, additional distribution of data events, and best-fit component likelihood of selected events. 